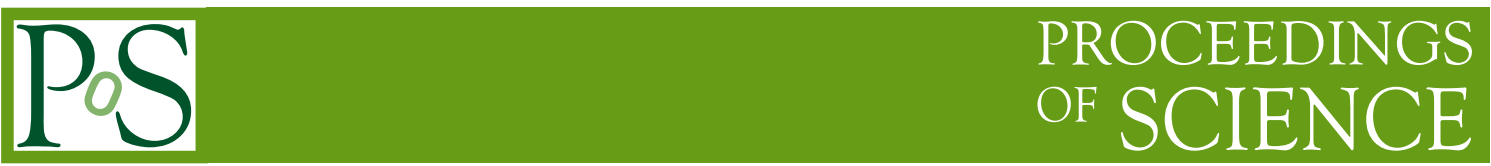

\title{
Gamma astronomy with the ARGO-YBJ experiment
}

\author{
Paolo Bernardini*, on behalf of the ARGO-YBJ Collaboration ${ }^{\dagger}$ \\ Dipartimento di Fisica, Università del Salento and INFN, Sezione di Lecce \\ via per Arnesano, 73100 Lecce, Italy \\ E-mail: paolo.bernardini@le.infn.it
}

\begin{abstract}
Very High Energy (VHE) $\gamma$-astronomy and cosmic ray physics are the main goals of the ARGOYBJ experiment. The detector is located in Tibet (People's Republic of China) and is a full coverage Extensive Air Shower array consisting of a carpet of Resistive Plate Chambers (RPCs). Altitude and full coverage ensure an energy threshold of few hundreds of $\mathrm{GeV}$ for primary photons detected in shower mode, while the RPC time resolution gives a good pointing accuracy, thus allowing a high sensitivity to $\gamma$-ray sources. The large field of view (FoV) and the high duty-cycle allow the continuous monitor of the Northern sky in the declination band $\left(-10^{\circ},+70^{\circ}\right)$. The detector operates also in scaler mode in order to look for Gamma Ray Burst (GRB) signals in the range 1-100 GeV in coincidence with GRB detection by satellites. After some detail about the performances of the detector, the present results concerning $\gamma$-astronomy will be presented.
\end{abstract}

25th Texas Symposium on Relativistic Astrophysics - TEXAS 2010

December 06-10, 2010

Heidelberg, Germany

* Speaker.

$\dagger$ This work is supported in China by NSFC (Contract No. 10120130794), the Chinese Ministry of Science and Technology, the Chinese Academy of Sciences, the Key Laboratory of Particle Astrophysics, CAS, and in Italy by the Istituto Nazionale di Fisica Nucleare (INFN) and the Ministero dell'Istruzione, dell'Università e della Ricerca (MIUR). 
The ARGO-YBJ (Astrophysical Radiation Ground-based Observatory at YangBaJing) experiment is located in Tibet at an altitude of $4300 \mathrm{~m}$ above sea level and is supported by an ItalianChinese scientific collaboration. It is mainly devoted to cosmic ray physics and gamma astronomy.

The altitude, the full coverage with proper space-time granularity, and the RPC time resolution allow an excellent reconstruction of the shower arrival direction. The FoV $(>2 s r)$ is limited only by the atmosphere thickness, the data-taking does not require particular environment conditions and the duty-cycle is very high. Therefore the continuous sky survey in the declination band from $-10^{\circ}$ to $+70^{\circ}$ is possible. Thanks to modularity the data-taking began during the commissioning, the central carpet was completed in 2006. Since November 2007 the experiment is fully installed and in stable data-taking.

Besides VHE gamma astronomy another goal of the experiment is to study cosmic rays in the range $1-300 \mathrm{TeV}$ and more. Many significant analyses have been completed or are under way about proton cross section [1], cosmic ray spectrum and anisotropies, antiproton flux and so on. Unprecented details on the shower front are available thanks to the high granularity and these informations can be crucial to test the hadron interaction models. The recently implemented charge read-out will allow the extension of these studies in the region close to the knee.

\section{Detector features and performance}

The detector consists of a single layer of RPCs operated in streamer mode [2], on a total area of about $110 \times 100 \mathrm{~m}^{2}$ (Fig. 1). The central carpet $\left(78 \times 74 \mathrm{~m}^{2}\right)$ is fully active and surrounded by a sampling ring with other $1000 \mathrm{~m}^{2}$ (20\% of the outer ring) equipped with RPCs. The array is divided in 153 clusters, each made by 12 RPCs with a dedicated Local Station for the DAQ. The digital read-out of the RPCs is performed by means of inductive strips $\left(6 \times 62 \mathrm{~cm}^{2}\right)$ well suited to detect small air showers. The pad is the fast-OR of 8 strips and defines the space-time pixel, with a time resolution better than $2 n s$ [3]. The timing calibration of the pads is performed periodically by means of an innovative software method [4] which does not require dedicated data-

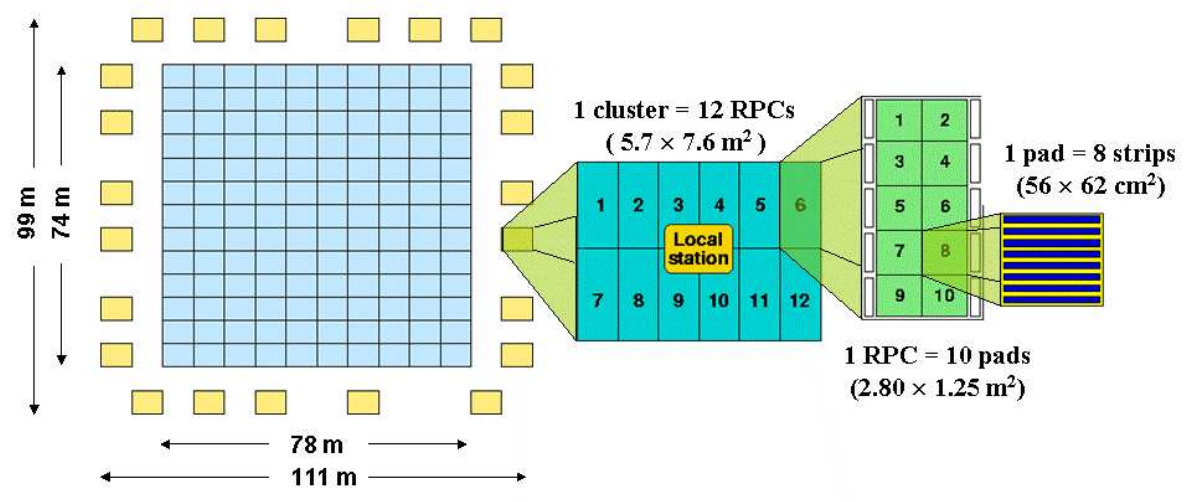

Figure 1: The set-up of the experiment. 

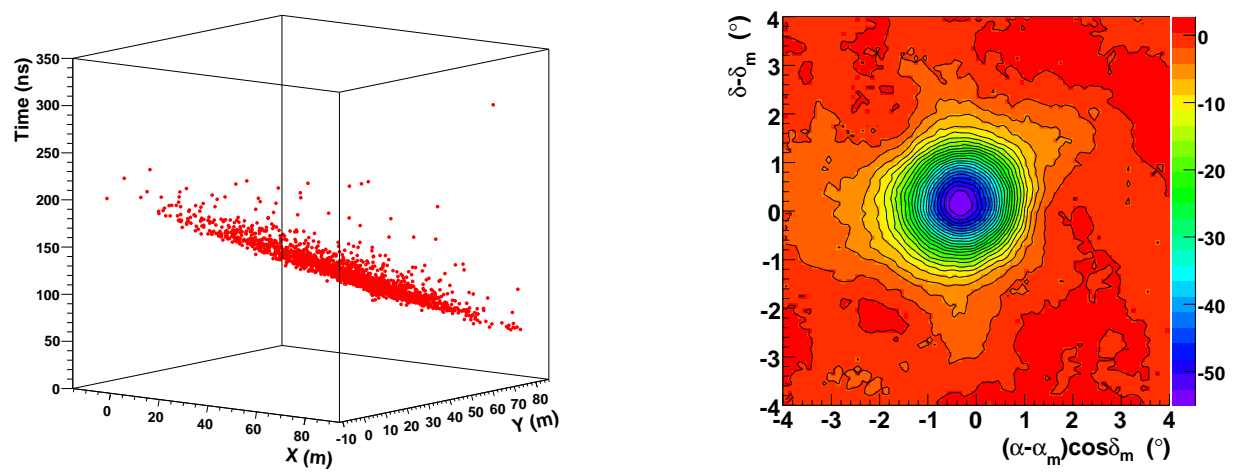

Figure 2: Left: An extensive air shower reconstruted by means of position and time of the fired pads. Right: The Moon shadow in the cosmic ray flux (significance map).

taking. Therefore the duty-cycle is limited only by few hardware maintenances and is higher than 85\%. In order to extend the measurable energy range, each RPC is equipped also with two large electrodes, called big pads, which provide an analog signal proportional to the deposited charge [5].

ARGO-YBJ collects data in shower and scaler mode. The first one works when the number of pads fired in a time-window of $420 n s$ exceeds the multiplicity (typically 20) required by the trigger condition. The energy threshold in shower mode is few hundreds of GeV. The features of the showers (arrival direction, core position, lateral distribution and so on) are fully reconstructed looking at the space-time pattern of the hit pads (Fig. 2, left). The scaler mode does not require any trigger, it records the rate for four multiplicities $(\geq 1, \geq 2, \geq 3$ and $\geq 4)$ on each cluster in a time window of $0.5 \mathrm{~s}$. The scaler mode is dedicated to study low energy $(E>1 \mathrm{GeV})$ transient phenomena (e.g. GRBs and solar flares) visible as non-statistical fluctuations of the background [6].

The Moon shadow (Fig. 2, right) in the cosmic ray flux is an irreplaceable check of the detector performances. The position and the intensity of the deficit are connected to the absolute pointing and to the angular resolution, respectively. Due to the geomagnetic field the cosmic rays are shifted toward West and this shift dependes on the rigidity, proportional to the ratio $E / Z$. Then the Moon shadow has been used to estimate the detector energy response. In Fig. 3 the angular resolution and the West-shift are shown versus hit multiplicity, in agreement with MonteCarlo simulation. In Fig. 4 the stability of the shadow measurement and of the small systematics $\left(\sim 0.2^{\circ}\right.$ North $)$ are shown. These data confirm the reliability of the detector and its stability.

\section{Search for Gamma Ray Bursts}

The data collected in scaler mode have been analysed to search for signals from GRBs in coincidence or with some delays with respect to the prompt emission detected by satellites. Presentely ARGO-YBJ analysed 102 bursts, the largest sample of GRBs on ground in the GeV range. Up to now statistically significant $\mathrm{GeV}$ signals of GRB counterpart were not detected and the fluence upper limits reached values as low as $10^{-5} \mathrm{erg} / \mathrm{cm}^{2}$ in the $1-100 \mathrm{GeV}$ range [7]. Also the shower mode data have been used in a stacked analysis to put significant upper limits on the GRB fluence up to $1 \mathrm{TeV}$ [8]. 

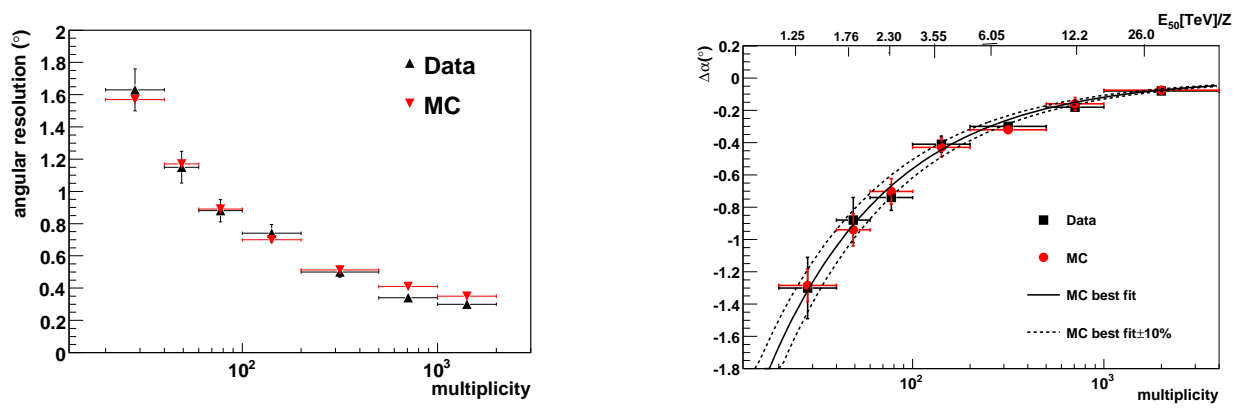

Figure 3: Moon shadow (data and simulation). Left: Angular resolution as a function of the hit multiplicity. Right: Westward shift of the Moon shadow due to the geomagnetic field as a function of hit multiplicity (the scale of the median energy over $Z$ is also shown).
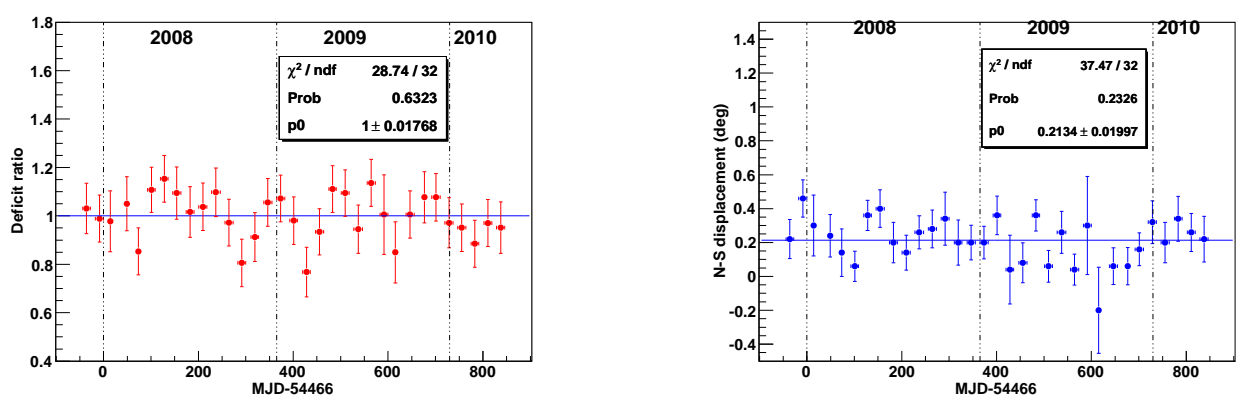

Figure 4: Moon shadow (more than two years of data). Left: Ratio between expected and measured deficit versus time. Right: Systematic North offset of the Moon shadow versus time.

\section{Gamma astronomy}

The search of $\gamma$-sources is performed using the data collected in shower mode. According to the Moon shadow analysis the detector Point Spread Function (PSF) is Gaussian for $N_{\text {hit }} \geq$ 100 while an additional Gaussian is required to describe the PSF at lower multiplicities. The angular resolution for cosmic ray showers is $\sim 1.6^{\circ}, 0.82^{\circ}$ and $0.66^{\circ}$ for $N_{\text {hit }} \geq 40,100$ and 300, respectively. By means of proper simulations the angular resolution for $\gamma$-induced showers has been evaluated $30-40 \%$ better because of the more defined profile of the showers. The background is calculated with two different methods (time swapping and equizenith) with consistent results.

With the data analysed up to now 3 sources have been identified (Crab Nebula, Markarian 421, MGRO J1908+06) and the detection sensitivity is around 0.5 Crab unit in one year. Other hot spots are going to become statistically significant collecting more data. Sensitivity enhancement and new results are also expected thanks to more sophisticated analyses (mainly gamma/hadron separation) in progress. Furthermore the continuous data taking in a large FoV allowed the observation of transient gamma-phenomena which the Cherenkov telescopes were not in condition to see. 

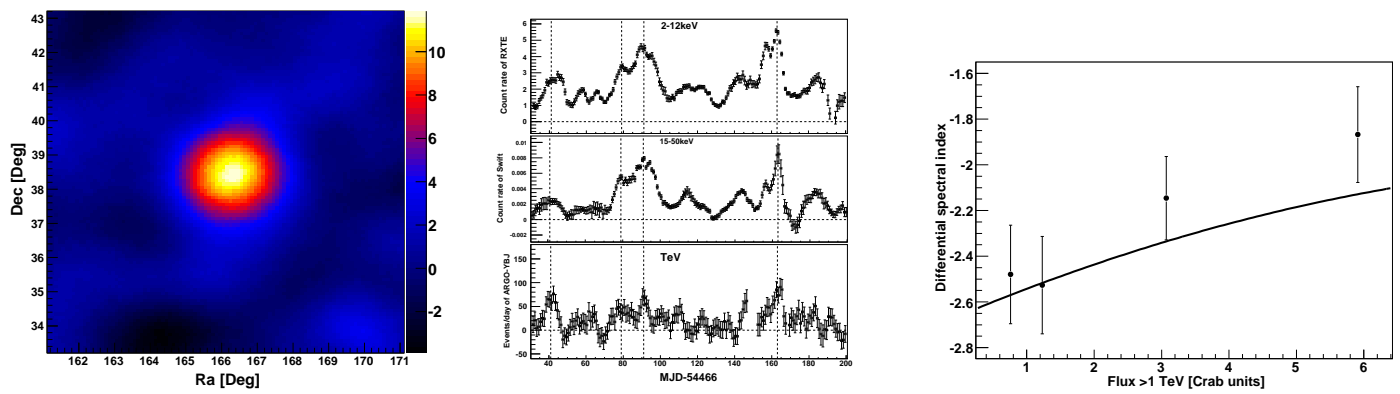

Figure 5: Mrk 421. Left: Significance map. Center: Light curve in different energy bands according to ASM/RXTE, BAT/Swift and ARGO-YBJ, respectively. Right: Spectral index as a function of gamma flux. The solid line is the function suggested by the Whipple experiment [13].

\subsection{Markarian 421 (Mrk 421)}

This Active Galatic Nucleus (AGN) is the first source detected by ARGO-YBJ (Fig. 5, left). During the flare in July and August $2006\left(E_{\gamma}>0.8 \mathrm{TeV}\right)$ the measured flux was 4 times higher than the Crab flux. In the first half of June 2008 another flare occurred, it has been studied from optical to $100 \mathrm{MeV} \gamma$-rays, and only partially up to $\mathrm{TeV}$ energies [9], since the moonlight hampered the Cherenkov telescope observations during the second and most intense phase of the emission. ARGO-YBJ data are available also during this second flare completing the multifrequency observations [10]. The signal intensity was about $6 \mathrm{Crab}$ units $\left(E_{\gamma}>1 \mathrm{TeV}\right)$ on June 11-13, with a significance of 4.2 standard deviations. In those days ARGO-YBJ measured the following spectrum

$$
d N / d E=(3.2 \pm 1.0) \times 10^{-11}(E / 2.5 \mathrm{TeV})^{-2.1 \pm 0.7} e^{-\tau(E)} \gamma / \mathrm{cm}^{2} / \mathrm{s} / \mathrm{TeV}
$$

where $e^{-\tau(E)}$ is the term due to Extragalactic Background Light [11]. This spectrum does not confirm the estimate by [9]. Its shape is consistent with the measurement by Whipple in 2000 and 2001 during a similar flare.

In February 2010 a third Mrk 421 flare was observed by ARGO-YBJ. We would like to stress that the data collected on February 16 were enough to see the source with a significance of almost $5 \sigma$. As we know this was the first time that an array observes a VHE gamma source just in one day.

A long monitor of Mrk 421 has been also performed [12] and the $\gamma$-signal shows a good correlation with X-ray emission (Fig. 5, center) without significant time delay. The TeV spectrum hardens when the flux increases (Fig. 5, right) according to Whipple [13]. This results are fully compatible with a $\gamma$-flux due to Synchrotron Self Compton (SSC) emission.

\subsection{MGRO J1908+06}

With 3-years data ARGO-YBJ observed (significance $>5 \sigma$ ) this source discovered by Milagro in 2007. The best ARGO-YBJ value for the source extension is $\sigma_{\text {ext }}=0.69^{\circ} \pm 0.25^{\circ}$. The measured spectrum is in agreement with the Milagro measurement, but only marginally consistent with HESS measurement, being the ARGO-YBJ flux a factor 3-4 higher. This result can be 

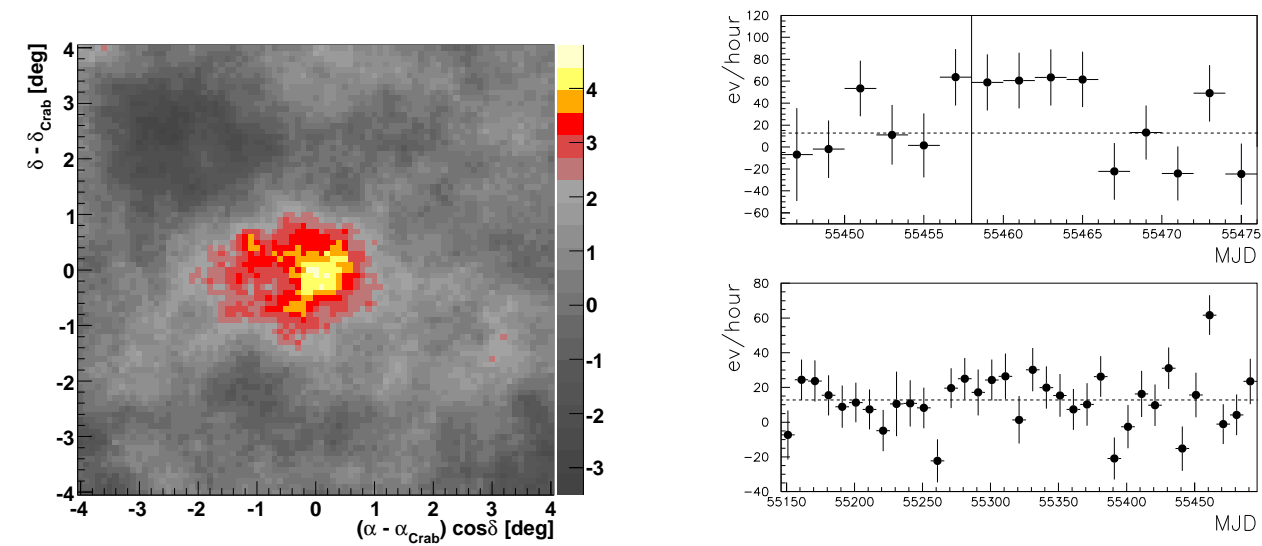

Figure 6: Crab Nebula. Left: Significance map in the flare days. Right: Light curves with 2-day bins (up) and with 10-day bins (down). The vertical line indicates the start time of the GeV-flare, the horizontal lines indicate the steady $\mathrm{TeV}$ emission.

explained by the worst angular resolution by Milagro and ARGO-YBJ with respect to HESS, assuming a signal contamination of other extended sources close to the object measured by the HESS telescope. Another possible explanation is a variability of the source. However a long-term monitor based on the ARGO-YBJ data has shown that the signal is consistent with a stable flux, on time scales ranging from few days to months.

\subsection{Crab Nebula}

ARGO-YBJ observed ( $\sim 14 \sigma$ in $\sim 800$ days) also the so-called "standard candle" of $\gamma$ astronomy. The measurement of the spectrum resulted fully compatible with those by other experiments:

$$
d N / d E=(3.62 \pm 0.29) \times 10^{-11}(E / T e V)^{-2.55 \pm 0.10} \mathrm{\gamma} / \mathrm{cm}^{2} / \mathrm{s} / \mathrm{TeV}
$$

In September 2010 an unexpected flare from Crab Nebula was detected by Agile and FermiLAT [14] in the band $0.1-10 \mathrm{GeV}$. The flare was visible also in the ARGO-YBJ data [15]. A flux increase by a factor of 3-4 compared to the non-flaring status has been detected both in the $\mathrm{GeV}$ and $\mathrm{TeV}$ range. Indeed ARGO-YBJ detected the source with a statistical significance of $4.8 \sigma$ (Fig. 6 , left) instead of $0.96 \sigma$ as expected in 8 days for the steady regime. The statistics does not allow to calculate the spectrum but the signal is essentially at low hit-multiplicities and then we can infer that the spectrum is soft (mainly $E_{\gamma}<1 \mathrm{TeV}$ ). The right plot of Fig. 6 shows the light-curve in the flare period with 10-day and 2-day bins. Since 2007 ARGO-YBJ never observed similar excesses. The probability that the 10-day excess is a fluctuation of the steady flux is $7 \times 10^{-6}$. Taking into account the trials the probability increases at $10^{-3}$.

Cherenkov telescopes did not confirm the ARGO-YBJ measurement [16]. Taking into account the different longitudes of the detectors and the 12-hours variability observed in the $\mathrm{GeV}$ range [17] this discrepancy can be explained assuming a similar variability at the $\mathrm{TeV}$ energy. 


\section{Conclusions}

The ARGO-YBJ detector is taking data since November 2007 in the complete setup (central carpet and guard ring) in a very stable way (the duty-cycle is more than $85 \%$ ). The detector performances are what expected and they are monitored by the analysis of the Moon shadow in the cosmic ray flux. Many remarkable results have been achieved in cosmic ray physics. Here the search for and the study of VHE gamma sources mainly based on two-year data have been presented. The Markarian 421 AGN has been continuously monitored and flares have been detected in 2006, 2008 and 2010. Comparing X and $\gamma$ data the SSC model results favorite for this source. The spectrum of MGRO J1908+06 has been measured in agreement with Milagro. The Crab Nebula has been observed in quiet emission and the spectrum has been measured according to other detectors. In September 2010 an unprecedented flare has been observed at $\mathrm{TeV}$ energies while satellite experiments detected it at $\mathrm{GeV}$ energy.

Many efforts are undergoing in order to increase the ARGO-YBJ sensitivity to gamma sources by means of data selection and $\gamma$-hadron separation. Presently ARGO-YBJ is the only wide-FoV $\gamma$-telescope able to monitor the sky day-by-day. The VHE gamma-sky survey is going on.

\section{References}

[1] G. Aielli et al (ARGO-YBJ Collaboration), Phys. Rev. D 80 (2009) 092004

[2] G. Aielli et al (ARGO-YBJ Collaboration), Nucl. Instrum. Methods A 562 (2006) 92

[3] G. Aielli et al (ARGO-YBJ Collaboration), Nucl. Instrum. Methods A 608 (2009) 246

[4] H.H. He et al, Astropart. Phys. 27 (2007) 528; G. Aielli et al (ARGO-YBJ Collaboration), Astropart. Phys. 30 (2009) 287

[5] G. Aielli et al (ARGO-YBJ Collaboration), to be published on Nucl. Instrum. Methods A

[6] G. Aielli et al (ARGO-YBJ Collaboration), Astropart. Phys. 30 (2008) 85

[7] G. Aielli et al (ARGO-YBJ Collaboration), Astrophysical Journal 699 (2009) 1281

[8] G. Aielli et al (ARGO-YBJ Collaboration), Astropart. Phys. 32 (2009) 47

[9] I. Donnarumma et al, Astrophysical Journal 691 (2008) L13

[10] G. Aielli et al (ARGO-YBJ Collaboration), Astrophysical Journal 714 (2010) L208

[11] M. Raue, D. Mazin, Int. J. Mod. Phys. D 17 (2008) 1515

[12] G. Aielli et al (ARGO-YBJ Collaboration), submitted to Astrophysical Journal

[13] F. Krennich et al, Astrophysical Journal 575 (2002) L9

[14] M. Tavani et al (AGILE Collaboration), Astronomer's Telegram 2855, September 22, 2010;

R. Buehler et al (FERMI-LAT Collaboration), Astronomer's Telegram 2861, September 23, 2010;

M. Tavani et al, Science 331 (2011) 736; A.A. Abdo et al, Science 331 (2011) 739

[15] G. Aielli et al (ARGO-YBJ Collaboration), Astronomer's Telegram 2921, October 11, 2010

[16] M. Mariotti (MAGIC Collaboration), Astronomer's Telegram 2967, October 23, 2010; R.A. Ong (VERITAS Collaboration), Astronomer's Telegram 2968, October 23, 2010

[17] M. Balbo et al, Astronomy \& Astrophysics 527 (2011) L4 